\title{
A Magnetic Field Response Recorder: A New Tool for Measurement Acquisition
}

\author{
Stanley E. Woodard ${ }^{*}$ and Bryant D. Taylor ${ }^{+}$ \\ *NASA Langley Research Center, Hampton, Virginia, U.S. A, Stanley.E.Woodard@nasa.gov \\ ${ }^{+}$Swales Corporation, Virginia, U.S. A, B.D.Taylor@larc.nasa.gov
}

\begin{abstract}
A magnetic field response recorder was developed to facilitate a measurement acquisition method that uses magnetic fields to power and to interrogate all sensors. Sensors are designed as electrically passive inductive-capacitive or passive inductive-capacitive-resistive circuits that produce magnetic field responses when electrically activated by oscillating magnetic fields. When electrically activated, the sensor's magnetic field response attributes (frequency, amplitude and bandwidth) correspond to the one or more physical states that each sensor measures. The response recorder makes it possible to simultaneously measure two unrelated physical properties using this class of sensors. The recorder is programmable allowing it to analyze one or more response attributes simultaneously. A single sensor design will be used to demonstrate that the acquisition method and the sensor example can be used to for all phases of a component's life from manufacturing to damage that can destroy it.
\end{abstract}

\section{Introduction}

The magnetic field response recorder, Fig 1, was developed to be a user friendly programmable self-contained portable unit to power and interrogate any passive inductive-capacitive sensor or passive inductive-capacitive-resistive sensor. This eliminates the shortcomings of many measurement acquisition systems such as the need for a sensor to have a physical connection to a power source and data acquisition hardware. Within a prescribed frequency range, the response recorder produces a sequence of magnetic fields with increasing harmonics frequencies that are used to power the sensors that in-turn respond with their own magnetic fields. It is this returned magnetic field what provides a measure of the physical properties being studied. This class of sensors is referred to as magnetic field response sensors. The response recorder eliminates the need for dedicated measurement channels and significantly reduces the logistics needed use sensors. Multiple sensors can be powered and interrogated using the same response recorder. However, each sensor must have a dedicated frequency partition of the antenna bandwidth and the interrogation system must be augmented with a measurement correlation table for each sensor. A goal in developing the recorder was to have a method to allow a single sensor to measure unrelated physical states and to achieve measurements in environments that would normally destroy electrical components. Use of the response recorder and the magnetic field response sensors also eliminates wire weight, wire degradation due to wear or chemical decay and the logistics needed to add new sensors. Eliminating wiring for acquiring measurements can alleviate potential hazards associated with wires such as damaged wires becoming ignition sources due to arcing. A detailed discussion of the acquisition method physics are given in Refs 1 and 2. 
Radio frequency identification (RFID) and other wireless sensing systems function as measurement telemetry systems. The sensor is part of an electrical circuit that includes a processor that is also connected to an antenna. The processor functions as a data acquisition system. Passive RFID systems are powered via an external single frequency oscillating magnetic field generated by the RFID reader. Active RFID systems use batteries. These wireless methods allow the measurements to be communicated via the antenna to other systems. ${ }^{3,4}$ The methods require that the circuit not be damaged else no measurement will be possible. Using the measurement system discussed in Ref. 1 facilitated by the magnetic field response recorder, the sensor is not placed in electrical connection with any processor or power source. More importantly, the system provides a means of acquiring measurements from a sensor when it is partially destroyed. Using the measurement system, partial destruction of wireless sensors is now being pursued as a measurement technique. There are three key improvements over current RFID and wireless sensor systems: (1) The system adds multi-functional capability to sensors-a single sensor can simultaneously measure two unrelated physical states (e.g., fluid level and position); (2) The sensors need no batteries and no wires for power or communications, so they can function in extreme environments; and (3) A sensor' measurement function can transform to suit changing sensing needs over the lifecycle of a component.

This paper presents an overview of the hardware and examples of the algorithms used to conduct the measurements. The response recorder has two key facets: The hardware for producing a varying magnetic field at a prescribed frequency and algorithms for controlling the magnetic field produced and analyzing sensor responses. Following the introduction, the response recorder hardware design will be presented followed by a multi-measurement sensor design. Measurements are then presented that show the potential for using the multi-measurement sensor design for tracking material phase transition, as a non-mechanical method of determining wheel speed and as means of identifying cracks or tears.

\section{The Magnetic Field Response Recorder Hardware Design}

The basic function of the response recorder is to transmit a magnetic field harmonic to power the sensor, switch to a receiving mode and then rectify and store the magnetic field response amplitude from the sensor. The frequency of the harmonic is also stored. For each harmonic, an antenna electrically coupled to an oscillating current (whose frequency is that of the harmonic) produces an oscillating magnetic field. Fig 2 shows the schematic of the hardware and control logic that facilitate transmission of the harmonic magnetic fields via an antenna and reception and interrogation of the magnetic fields that the sensors produce. The oscillating field produces an electromotive force in the sensor making the sensor electrically active. The sensor now produces its own oscillating magnetic field with the field frequency, amplitude and bandwidth dependent upon the physical properties being measured. Sensor responses to two series of sequentially transmitted harmonics are illustrated in Fig 3 for two sensor interrogations at times $t_{1}$ and $t_{2}$. Because the sensor is a resonant circuit, the response amplitude, $A_{i}$, is dependent upon the transmitted harmonic frequency. Each response is compared to the previous one, $\mathrm{A}_{\mathrm{i}-1}$, to determine if the peak response has been acquired. If the current amplitude is less than the stored amplitude then the stored amplitude is response peak amplitude. The "transmit-receivecompare" of sequential harmonics is repeated until the peak response is identified. Harmonic 
increments, $\Delta \omega_{\mathrm{i}}$, are provided by the user as part of sensor calibration data. The harmonic producing the peak amplitude is the sensor resonant frequency. The transmitted harmonic's frequency and the response amplitude are stored and then correlated to calibration data. ${ }^{1,2}$

The components of the recorder are a microcontroller, frequency synthesizer, internal antenna, connection for an external antenna, power supplied via batteries or external line voltage, a liquid crystal display, memory, low-pass filter, an analog-to-digital converter and a connection for an analog output. The schematic, Fig 2, illustrates the control logic and antenna signals during transmission and reception. During transmission, the microcontroller places an antenna into transmission mode and submits a binary code to the frequency synthesizer. The frequency corresponding to this code is stored. The synthesizer converts the code into a square wave whose frequency is dependent upon the code. A high-speed amplifier then amplifies the square wave. All frequencies that are higher than the prescribed frequency are then attenuated using a low pass filter. The signal is then applied to the antenna for a prescribed number of cycles of the wave. The signal to the antenna results in a time varying magnetic field. When the cycles are completed or after a set time duration is completed, the microcontroller switches the antenna to a receiving antenna. During the transmission, the magnetic field response sensor is being electrically excited. The sensor's magnetic field decays when the antenna is placed in the receiving mode. The response from the sensor is amplified after being received from the antenna. A diode peak detector rectifies the signal (i.e., only the positive value of the signal is allowed to pass) and creates a DC value proportional to signal amplitude (i.e., a capacitor charge is proportional to signal amplitude). An operational amplifier (op-amp) amplifies the DC voltage from the peak detector. The signal from the op-amp is then converted into a digital signal. The microprocessor stores the amplitude (digital signal from op amp) and the transmission frequency. The amplitude and frequency can be used for further analysis of the physical states by user supplied programs to the microcontroller.

\section{A Multi-Measurement Sensor Design}

The response recorder can power and analyze the response from any inductive-capacitive or inductive-capacitive-resistive circuit that produces a harmonic magnetic field when it resonates. The frequency response peak amplitude, resonant frequency and bandwidth are dependent upon the circuits intrinsic properties (e.g., resistance), energy applied and/or removed, geometric changes to the circuit that alter its capacitance or inductance, or, environmental changes that alter the circuits inductance, capacitance or resistance. A traditional measurement acquisition system acquires only one variation in a sensor's circuit (e.g., voltage) as a physical state changes. Correction tables are used to account for other influences on the circuit (e.g., temperature).

A novel feature of the response recorder is that more than one physical state can be simultaneously measured using a single magnetic field response sensor. This is achieved by correlating combinations of different physical states (e.g., strain, out-of-plane displacement) to combinations of different sensor response attributes (frequency, amplitude, respectively). The sensor can be used to measure a permanent change of state (e.g., curing, material phase 
transition, chemical reaction) before other measurement uses. Once the new physical state has been achieved, the sensor can simultaneously measure other physical states.

When a sensor measures a single physical state, a calibration curve is used to correlate an electrically varied quantity (e.g., sensor voltage) to a physical state. When two independent physical states are measured simultaneously using the same magnetic field response sensor, it is necessary to correlate a surface formed from combinations of two response attributes (response surface) to a surface formed from combinations of physical states (state surface). Fig 4 illustrates a state surface and a response surface. Points are annotated on the state surface for three combinations of state 1 and state 2 . The corresponding response points are annotated on the response surface. The surfaces are valid for measurement if each point of the state surface correlates to only one point on the response surface. The extrema of the surfaces do not have to be the same. For example, the minimum of state 1 and minimum of state 2 need not to correlate to the minima of response attribute 1 and response attribute 2 but strict one-to-one mapping is required. In practical applications, discrete points on the state surface are measured to identify the corresponding points on the response surface. The resolution of points is dependent upon the desired measurement resolution. Table 1 shows a table of points used for calibration. The table allows combinations of amplitude and frequency to be correlated to combinations of physical values for state $\mathrm{X}$ and state $\mathrm{Y}$. For example, element $(3,3)$ correlates amplitude, $\mathrm{A}_{3}$, and frequency, $\omega_{3}$, to the values $\mathrm{X}_{3}$ and $\mathrm{Y}_{3}$ for states $\mathrm{X}$ and $\mathrm{Y}$, respectively.

\begin{tabular}{|l|l|l|l|l|l|l|}
\hline & & \multicolumn{5}{|c|}{ Variation of Physical State $\mathrm{Y}$} \\
\cline { 3 - 7 } & & \multicolumn{1}{|c|}{$\mathrm{Y}_{1}$} & \multicolumn{1}{c|}{$\mathrm{Y}_{2}$} & \multicolumn{1}{c|}{$\mathrm{Y}_{3}$} & $\mathrm{Y}_{4}$ & $\mathrm{Y}_{5}$ \\
\hline \multirow{4}{*}{$\begin{array}{l}\text { Variation of } \\
\text { Physical State }\end{array}$} & $\mathrm{X}_{1}$ & $\mathrm{~A}_{1}, \omega_{1}$ & $\mathrm{~A}_{2}, \omega_{1}$ & $\mathrm{~A}_{3}, \omega_{1}$ & $\mathrm{~A}_{4}, \omega_{1}$ & $\mathrm{~A}_{5}, \omega_{1}$ \\
\cline { 2 - 7 } & $\mathrm{X}_{2}$ & $\mathrm{~A}_{1}, \omega_{2}$ & $\mathrm{~A}_{2}, \omega_{2}$ & $\mathrm{~A}_{3}, \omega_{2}$ & $\mathrm{~A}_{4}, \omega_{2}$ & $\mathrm{~A}_{5}, \omega_{2}$ \\
\cline { 2 - 7 } & $\mathrm{X}_{3}$ & $\mathrm{~A}_{1}, \omega_{3}$ & $\mathrm{~A}_{2}, \omega_{3}$ & $\mathrm{~A}_{3}, \omega_{3}$ & $\mathrm{~A}_{4}, \omega_{3}$ & $\mathrm{~A}_{5}, \omega_{3}$ \\
\cline { 2 - 7 } & $\mathrm{X}_{4}$ & $\mathrm{~A}_{-1}, \omega_{4}$ & $\mathrm{~A}_{-2}, \omega_{4}$ & $\mathrm{~A}_{3}, \omega_{4}$ & $\mathrm{~A}_{4}, \omega_{4}$ & $\mathrm{~A}_{5}, \omega_{4}$ \\
\cline { 2 - 7 } & $\mathrm{X}_{5}$ & $\mathrm{~A}_{1}, \omega_{5}$ & $\mathrm{~A}_{2}, \omega_{5}$ & $\mathrm{~A}_{3}, \omega_{5}$ & $\mathrm{~A}_{4}, \omega_{5}$ & $\mathrm{~A}_{5}, \omega_{5}$ \\
\hline
\end{tabular}

Table 1 Multi-measurement sensor correlation table

The response surface allows one to relate the sensor response to phenomena being measured. In addition, it is use to help designers determine which measurements can be done independently or simultaneously using the same sensor. Similarly to the two dimensional response surface example a three-dimensional space could also be developed that correlates all response attributes to physical states. An example of a multi-measurement sensor will be used to demonstrate how the response surface can be used with the recorder.

An example of a multi-measurement sensor shown in Fig 5. Eleven sets of interdigitated electrodes are distributed along the length of the sensor and are electrically connected to an inductor creating a resonant circuit. The sensor is designed to function as long as at least one set of electrodes is electrically connected to the inductor. The sensor resonant frequency depends on the number of electrode sets electrically connected to the inductor. Fig 6 shows how the normalized resonant frequencies vary as the number of active electrode sets change from 1 to 10 sets of electrodes. The frequencies are normalized to the frequency of the circuit 
with a single set of electrodes. If the sensor initially has 10 active sets of electrodes, its frequency is approximately 0.30 that of a sensor with only one set. The sensor is designed to work with a damage electrode sets if there is a crack or tear along the sensor. Hence, its undamaged frequency is 0.30 that of the inductor connected to a single set of that it can be damaged. If a crack occurs along the sensor between any sets of electrodes, a frequency shift will occur in the sensor.

Measurements from individual magnetic field response sensors can be used to infer that the sensor shown in Fig 5 can be used for measuring the formation of a component (curing and material phase transition), use of the component (displacement) and damage to the component (tearing or cracking). 


\section{Material Phase Transformation}

Figure 7 shows the magnetic field response sensor used to measure material phase transition. The inductor is formed as a square spiral trace of copper. Interdigitated electrodes have been used for the capacitor. The inductor and the capacitor have been deposited on a thin dielectric film. Three sensors were placed at the bottom of plastic containers. Liquid resin was poured into the containers and became a dielectric of the capacitors resulting in the sensor resonant frequencies changing. Time histories of the sensor amplitudes and frequencies are shown in Fig 8. As the resin cured, its dielectric constant changed resulting in a changed capacitive value of the L-C ciruit. As seen in Fig. 8, the response frequencies no longer changed after approximately 200 hours indicating that the curing was complete.

To monitor curing, the response recorder could be programed with a simple problem with the following control logic

\section{While}

Then

$$
\begin{aligned}
& \left(\omega\left(t_{i}\right)-\omega\left(t_{i-1}\right)\right) \text { not equal to } 0 \\
& \text { and } \\
& \left(A\left(t_{i}\right)-A\left(t_{i-1}\right)\right) \text { not equal to } 0
\end{aligned}
$$

\section{Component curing is not complete Store $\omega\left(t_{i}\right)$ and $A\left(t_{i}\right)$}

$\boldsymbol{A}\left(\boldsymbol{t}_{i}\right)$ and $\boldsymbol{\omega}\left(\boldsymbol{t}_{i}\right)$ are the sensor amplitude and frequency, respectively at $\boldsymbol{t}_{\boldsymbol{i}}$. The program tracks the curing process and collects the frequencies and amplitudes to identify what portion of the response surface is needed for tracking the curing process. The response trajectory of the curing process for the three sensors is shown in Fig 9. Should another measurement be desired using the sensors while they are be cured, it would be possible to do so as long the combinations of amplitude and frequencies did not overlap the amplitude-frequency trajectory shown in Fig 9. Other measurements could also be achieved during curing if the other dimension of the response space (bandwidth) is used. After the sensors are cured, they could be calibrated and used for other measurements. One such measurement could be displacement of a component. 


\section{Displacement and Non-Mechanical Wheel Speed Sensor}

The previous sensor example was that of sensors placed in a resin. As the resin cured, the response amplitudes and frequencies changed. If the same sensor shown in Fig 5 is suspended in the mold of a tire that is surrounded by the rubber as the rubber fills the mold, it will track the rubber curing, as shown in the resin curing example. Once cured, it can be used as a a displacement or wheel speed sensor.

For displacement/rotation measurements, the response recorder antenna orientation and position are kept fixed. The sensor can move relative to the antenna but with a fixed orientation. The method requires calibration of response amplitude with distance from antenna. Using a constant sampling period, $\Delta \mathrm{t}$, the displacement rate $\mathrm{v}$ is computed as

$$
\mathrm{v}=\left[\left(A_{1}\left(t_{1}\right)\right)-\left(A_{1}\left(t_{2}\right)\right)\right] / \Delta \mathrm{t}
$$

A sensor with fixed frequency and fixed orientation with respect to the response recorder antenna can be used for position and displacement measurements. Sensor response amplitude is dependent upon inductor distance from antenna. If the sensor's orientation is not fixed but its trajectory is known, it may be possible to calibrate the response amplitude variation with trajectory. Rotation speeds of a wheel or propeller are examples where response amplitude can be used to provide a useful measurement with a sensor that does not maintain a fixed orientation.

An example of the necessary programming logic for the response recorder to measure wheel speed is

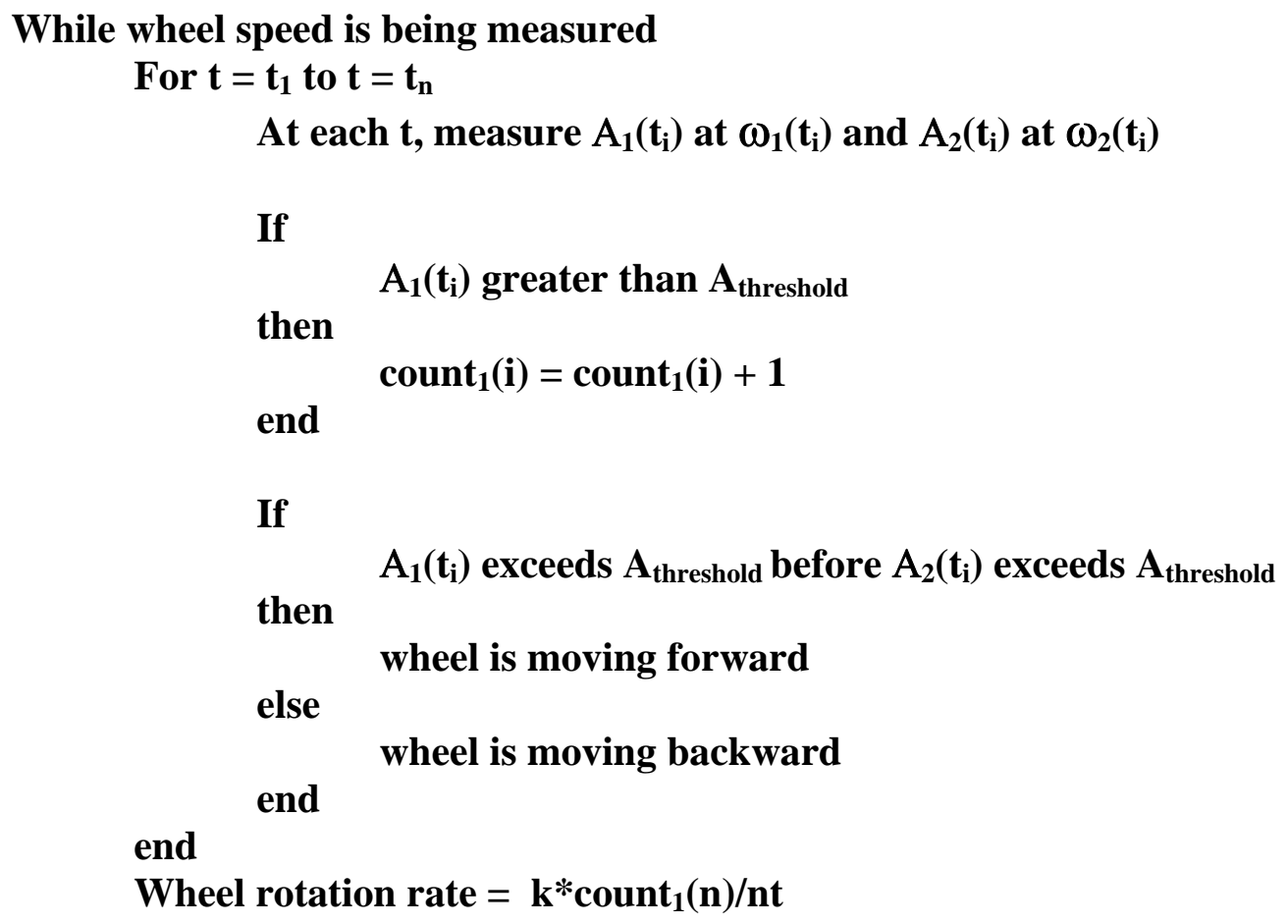


$\mathbf{A}_{\text {threshold, }}$ is the threshold amplitude; count $(\mathbf{i})$ is counter of time the amplitude has been exceeded, $\mathbf{A}_{\mathbf{1}}\left(\mathbf{t}_{\mathbf{i}}\right)$ and $\mathbf{A}_{\mathbf{2}}\left(\mathbf{t}_{\mathbf{i}}\right)$ are amplitudes of sensors 1 and 2, respectively at $\mathbf{t}_{\mathbf{i}}, \boldsymbol{\omega}_{\mathbf{1}}\left(\mathbf{t}_{\mathbf{i}}\right)$ and $\boldsymbol{\omega}_{\mathbf{2}}\left(\mathbf{t}_{\mathbf{i}}\right)$ are frequencies of sensors 1 and 2, respectively and, $\mathbf{k}$ is a constant that converts the count rate to a desired angular measurement such as kilometers per hour.

For rotational motion (e.g., propeller, wheel, etc), the sensor moves relative to the antenna but its orientation varies with position. Identifying the numbers of time the response amplitude exceeds a threshold amplitude in a fixed duration can be used to determine rotation rate. As the sensor approaches the antenna, the amplitude increases. The amplitude peaks at the closest point to the antenna then decreases producing changes in amplitude that are cyclical. A counter is used to track the number of times the threshold value is initially exceeded as the inductor approaches the antenna; number of counts in a given time interval is the rotation speed. For example, 10 counts in $1 \mathrm{~s}$ is a $10 \mathrm{~Hz}$ rotation. When two sensors are used inside the wheel, rotation direction can be determined by identifying which sensor amplitude increases first.

To demonstrate wheel speed measurements, two thin-film magnetic response sensors are placed 55 deg apart inside a wheel, as shown in Fig 10, with a pre-set fixed frequency of 5.8 and 6.4 $\mathrm{MHz}$. When incorporated into aircraft or automobiles tires, the method provides a way to determine wheel speed without additional sensors. A tire with sensors on, the response recorder, and the antenna are shown in Fig 11 with the sensor locations annotated. A laser is positioned on the wheel projects its light on wall in front of it. The wall has had marks placed on it to correspond to 1 degree increments so that the laser light position can be used to increment wheel rotation. In practical use, the antenna can be placed on or embedded in the wheel well. Note that unlike other commercial wheel speed sensors that are exposed to ambient conditions, the wheel serves as a protective housing for the sensor. The response from each sensor as the wheel is rotated is shown in Fig 12. As the wheel rotates, each response amplitude will grow until it reaches its peak at each sensor's closest approach to the antenna. Two sensors are used to discern rotation direction which is determined by which sensor's amplitude rises first.

The wheel speed measurement is a simple, inexpensive and a non-mechanical to measure rotation speed forward and backwards. This system presented in this section does not wear out because no gears are needed to provide a measurement using the Hall effect such as is being done commercially and the system antenna and sensor are extremely lightweight. The interrogation system can also be made light weight and have a smaller volume. Also, the sensor is not exposed to ambient conditions and the wheel serves as protective housing for sensor. 


\title{
Example of a Crack/Tear Detection Sensor
}

A new class measurement that the multi-measurement sensor shown Fig 5, will demonstrate is to identify whether it or more specifically, the surface it has been adhered or deposited upon has been torn or cracked. This function of the sensor can be used concurrent with other measurements that correspond to response bandwidth or amplitude (e.g., rotation) because it results in a frequency shift. The sensor can be placed on either a surface of a non-conductive material or can be deposited on each ply of a layered non-conductive composite material. Metal deposition techniques such as vapor deposition can be used to deposit the sensor directly on the surface. The deposited circuit has no structural strength to prevent breaking if the substrate breaks or cracks. Circuit thickness is approx 50 microns.

The capacitive component of the sensor is shown in Fig 13a. Fig 13b shows the capacitive interdigital electrodes are grouped into sets. The sets are distributed along the length of the sensor. Should a crack occur between the sets, a discrete change in capacitance will result and thus a discrete change in response frequency. The interdigitated electrodes (capacitor) are electrically connected to an inductor. When placed on a panel, the in-board set of electrodes should not be placed on panel. This will allow for a frequency response even if a crack exists on panel.

The sensor allows for spatial identification of crack/damage location along length of sensor. A crack change the number of electrically active interdigitated electrode sets resulting in a new frequency that is dependent upon the crack location along the sensor. Multiple sensors can be used to produce a 2-D profile of a crack. When used on multiple surfaces, the method allows for 3-D identification of crack profile.

During a material phase transition, the multi-measurement sensor can be used to monitor the transition. The frequency-amplitude trajectory on the response surface during a phase transition for resin curing is shown in Fig 9. This trajectory can be monitored using the response recorder and subsequently the sensor can also be used to monitor wheel rotation (amplitude variation with time) and cracks (frequency shift). The frequency-amplitude trajectory for wheel rotation is shown in Fig 14 for a single multi-measurement sensor. If the sensor has not been torn, the trajectory is repetitive along a single frequency. Should damage occur the trajectory is still repetitive along a single frequency but the frequency is now shifted from the undamaged specimen. The amount of the frequency shift is dependent upon where the damage on the sensor occurred.

To measure rotation while identification of wheel damage along sensor, the following logic could be programmed into the response recorder.

\author{
While wheel speed is being measured \\ For $t=t_{1}$ to $t=t_{n}$ \\ At each $t$, measure $A_{1}\left(t_{i}\right)$ at $\omega_{1}\left(t_{i}\right)$ and $A_{2}\left(t_{i}\right)$ at $\omega_{2}\left(t_{i}\right)$ \\ if
}




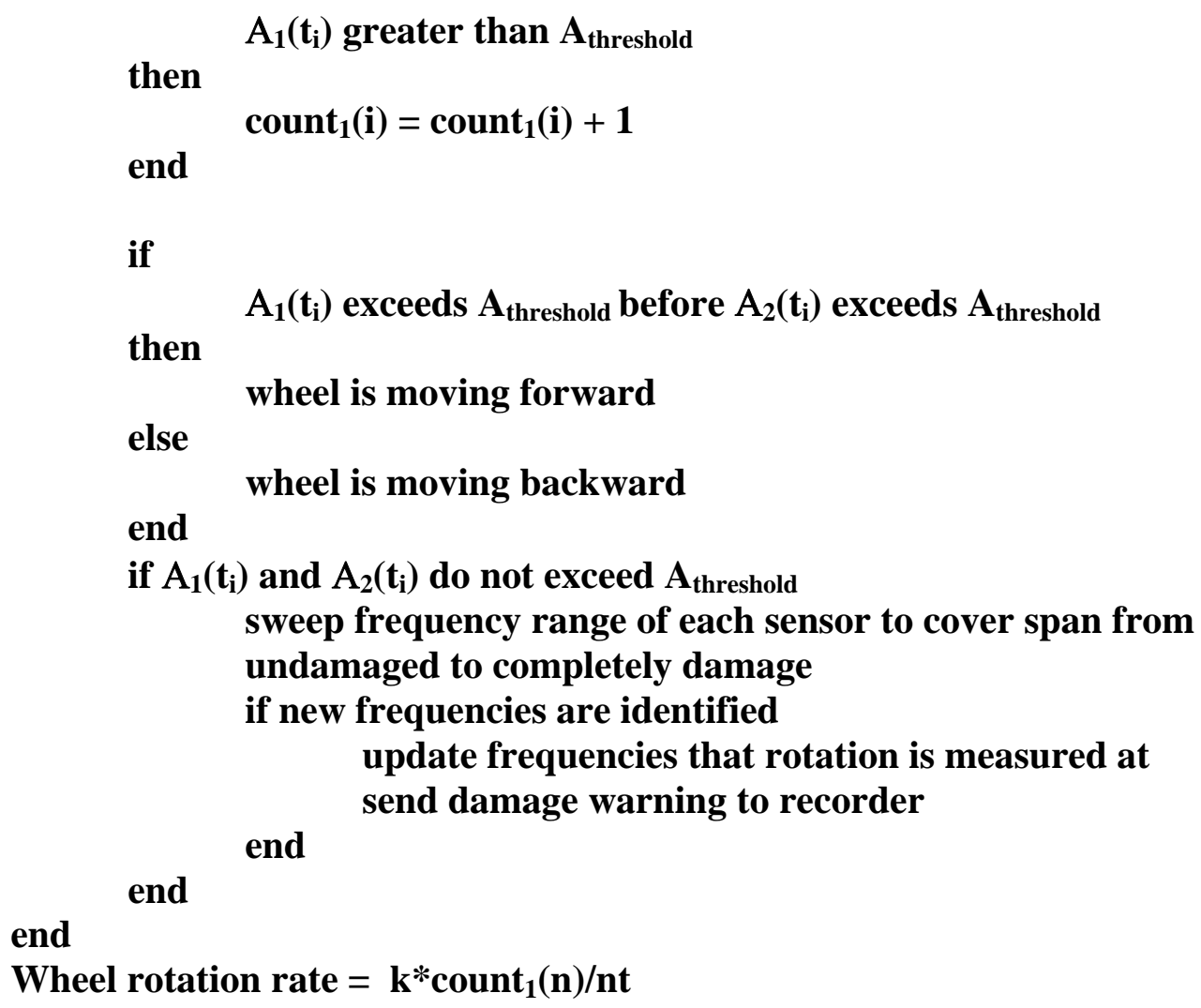

Other applications for concurrent rotation measurement and damage detection are as a noncontact non visual method of identifying package tampering; as a means of identifying damage/cracks to moving surfaces such as a pulley belt (e.g., fan belt) or within a vehicle tire. Table 2 shows possible individual and simultaneous measurements using the multi-measurement sensor. 


\begin{tabular}{|l|c|c|c|}
\hline & $\begin{array}{l}\text { Dielectric Change } \\
\text { from Phase } \\
\text { transition/Cure } \\
\text { Monitoring }\end{array}$ & $\begin{array}{l}\text { Displacement/ } \\
\text { Rotation }\end{array}$ & $\begin{array}{l}\text { Electrode Sets } \\
\text { Severed }\end{array}$ \\
\hline Wheels & $\mathrm{x}$ & $\mathrm{x}$ & $\mathrm{x}$ \\
\hline Pulleys & $\mathrm{x}$ & $\mathrm{x}$ & $\mathrm{x}$ \\
\hline Cement & & & $\mathrm{x}$ \\
\hline Tamper & & $\mathrm{x}$ & $\mathrm{x}$ \\
\hline Component motion & & & \\
\hline
\end{tabular}

Table 2 Individual and simultaneous measurements using the multi-measurement sensor.

\section{Conclusions}

A user friendly portable programmable measurement acquisition tool has been presented that can power and interrogate any passive inductive-capacitive sensor or passive inductive-capacitiveresistive sensor. The response recorder uses a sequence of oscillating magnetic fields to power the sensors and thus no physical contact is needed between the response recorder and the sensor. After the sensors are activated by the impinging magnetic field they produce a magnetic response whose attributes correspond to one or more measured physical states. To measure a single physical state, a calibration curve is developed between the increments of the physical state and the resulting response attribute. Simultaneous measurements require a two dimension mapping of combinations of physical states (i.e., a state surface) to the corresponding combinations of response attributes (i.e., a response surface). By using a response surface mapping one can relate multiple independent measurements of the same sensor to physical changes in the object being measured.

The tool - the magnetic field response recorder - eliminates the need for dedicated measurement channels and significantly reduces the logistics needed for using sensors. To show the capability of the response recorder, a multi-measurement sensor was produced. The sensor is capable of functioning when partially destroyed as long as a portion of it is still capable of resonating. The multi-measurement sensor can be used to measure key but unrelated physical states for three phases of a component's life - fabrication, use and damage that could possibly destroy a component. Even in cases where the sensor was progressively severred the design was capable of functioning partially damaged. Measurement results from three individual sensors were presented to show that these measurements are possible and that they could be reduced to a single sensor. For all measurements described, simple logic was that could easily be programmed into the recorder was presented to show that the unit could provide analysis of acquired measurements and to identify any changes to the sensor. 


\section{References}

1 S. E.Woodard, B. D. Taylor, Q. A. Shams and R. L. Fox, Magnetic Field Response Measurement Acquisition System, NASA Technical Memorandum 2005-213518, (2005).

2 S. E. Woodard and B. D. Taylor, A Wireless Fluid-Level Measurement Technique, NASA Technical Memorandum 2006-19271, (2006).

3. Raza, N., Bradshaw, V., Hague, M., “Applications of RFID technology,” RFID Technology (Ref. No. 1999/123), IEE Colloquium, 25 Oct. 1999.

4. Nath, B., Reynolds, F. and Want, R., "RFID Technology and Applications,” IEEE Pervasive Computing, Vol. 5, No. 1, pp. 22-24, Jan-Mar, 2006. 


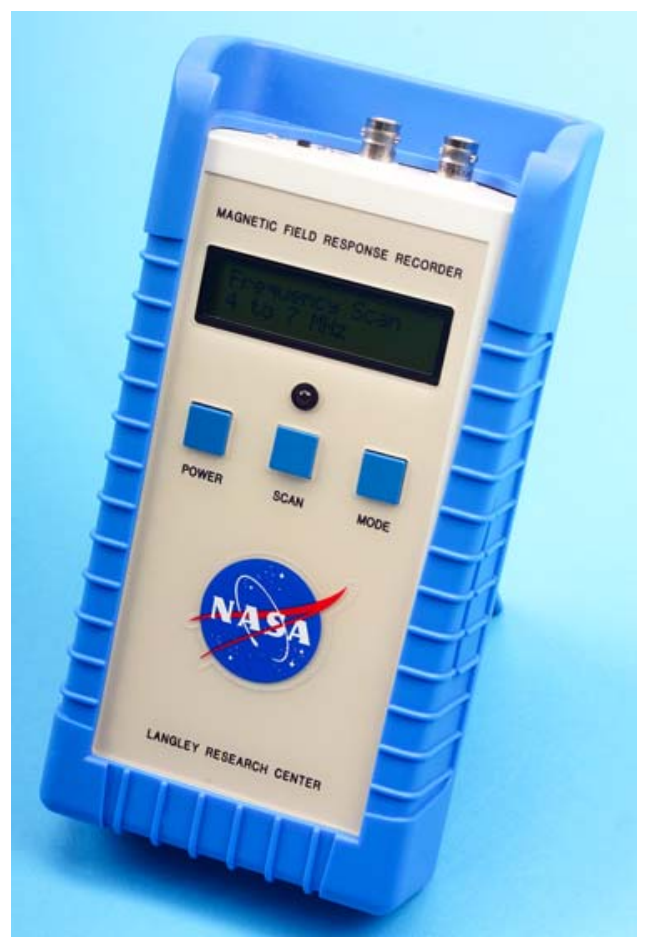

Fig 1 Magnetic field response recorder

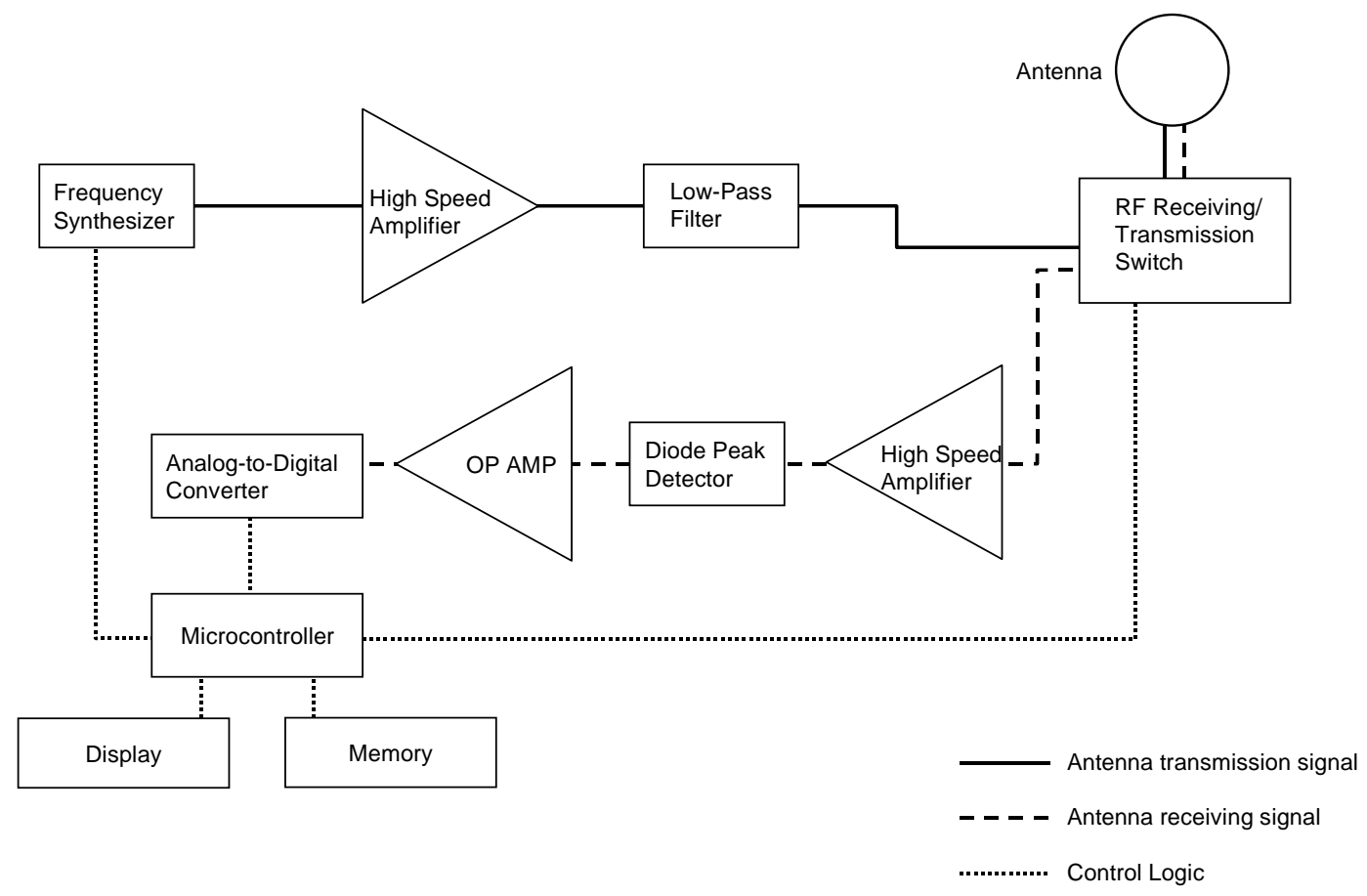

Fig 2 Schematic of control logic and antenna signals during transmission and reception. 


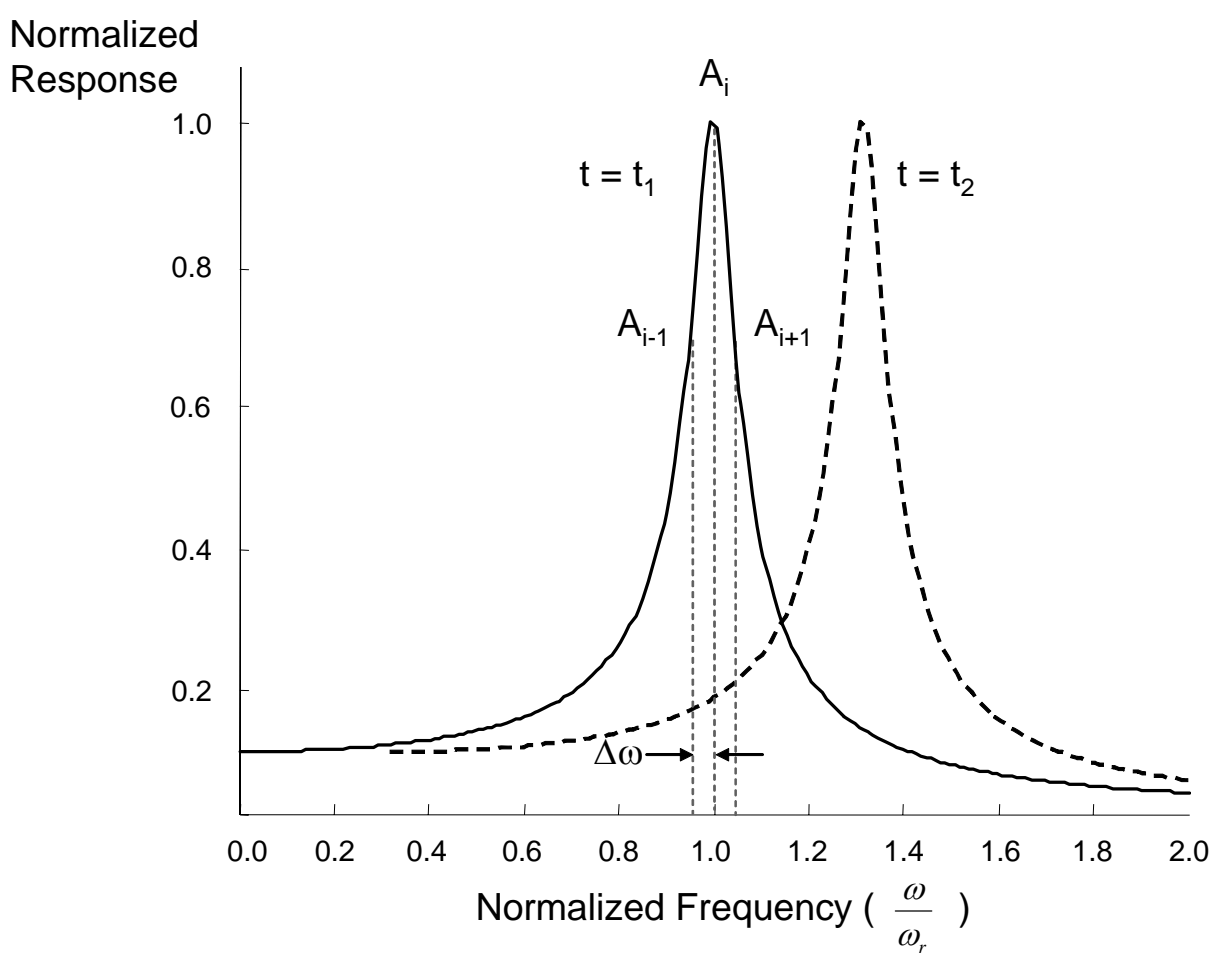

Fig 3 Sensor magnetic field response at two times

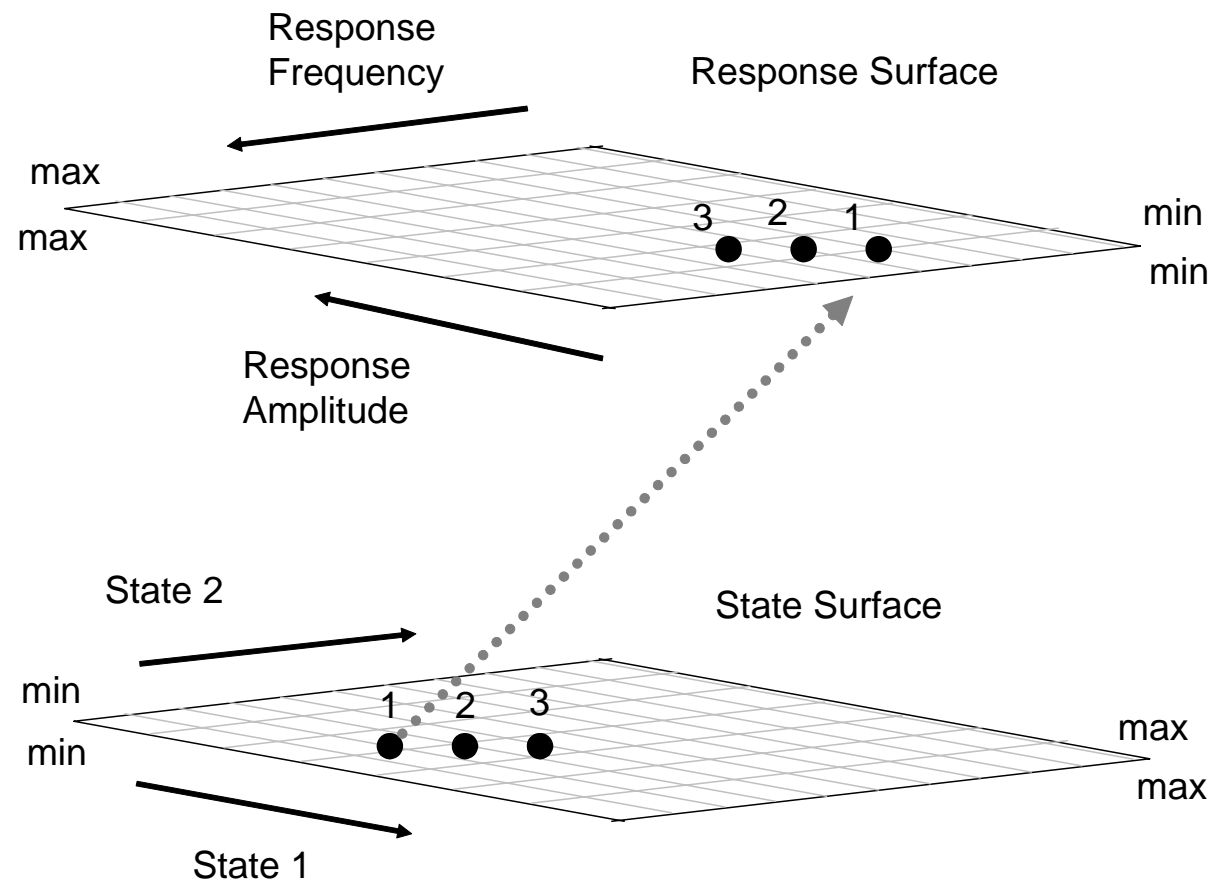

Fig 4 State surface and response surface. 

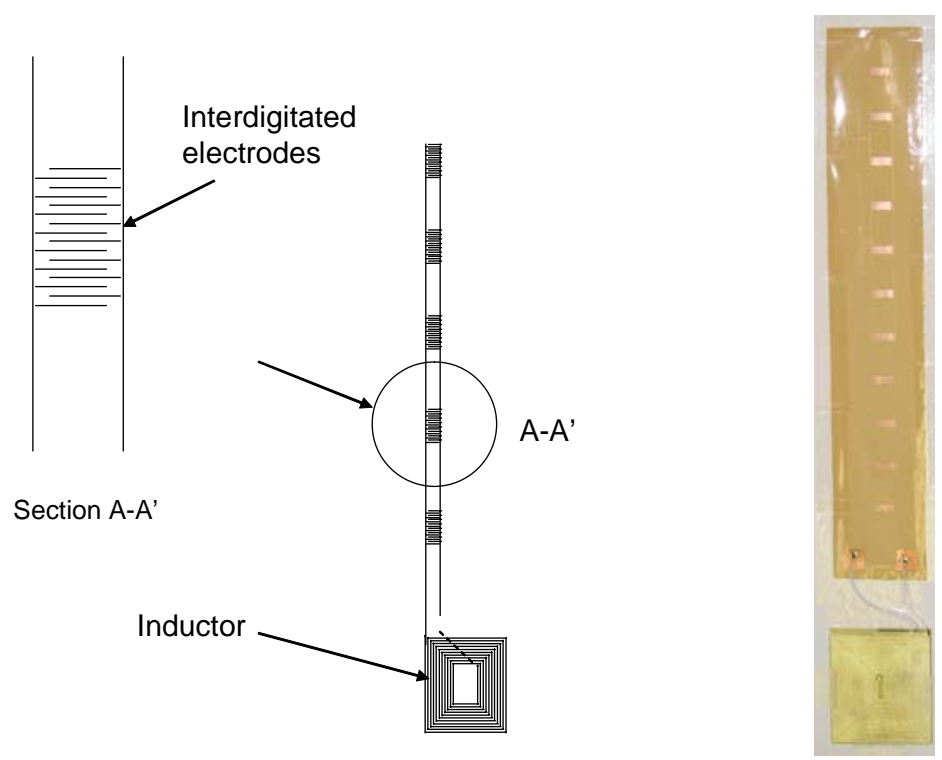

a. Sensor schematic

b. Sensor

Fig 5 Multi-measurement sensor

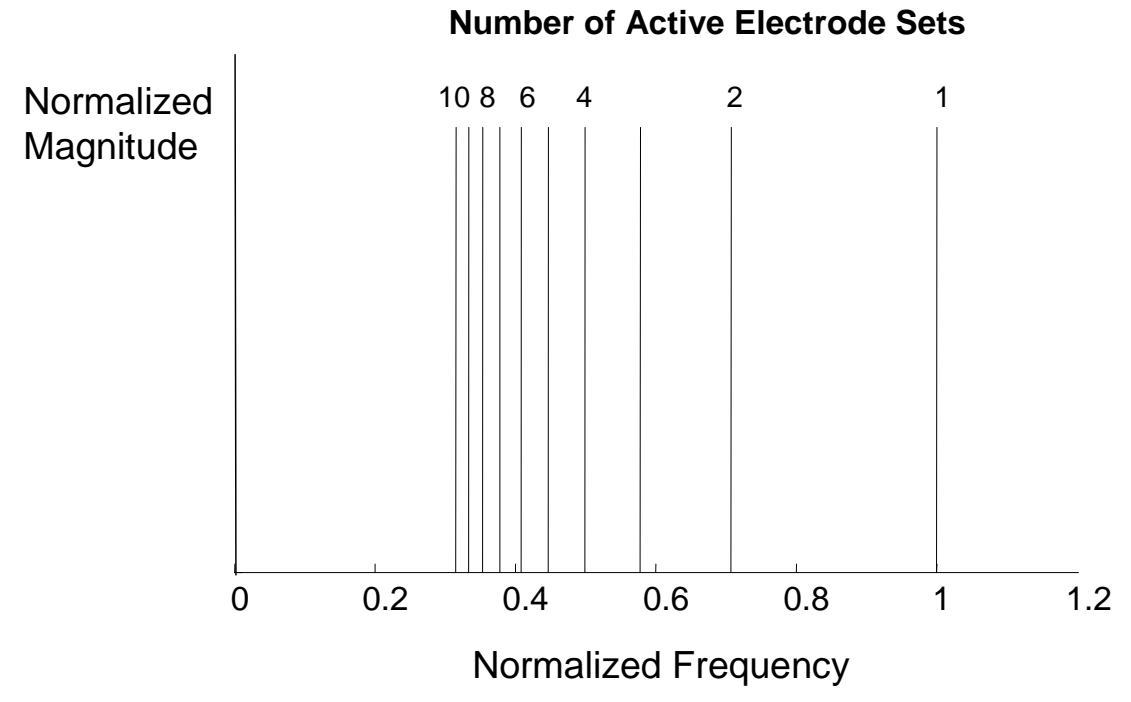

Fig 6 Normalized frequency scaled to resonant frequency of sensor with one interdigital capacitor set 


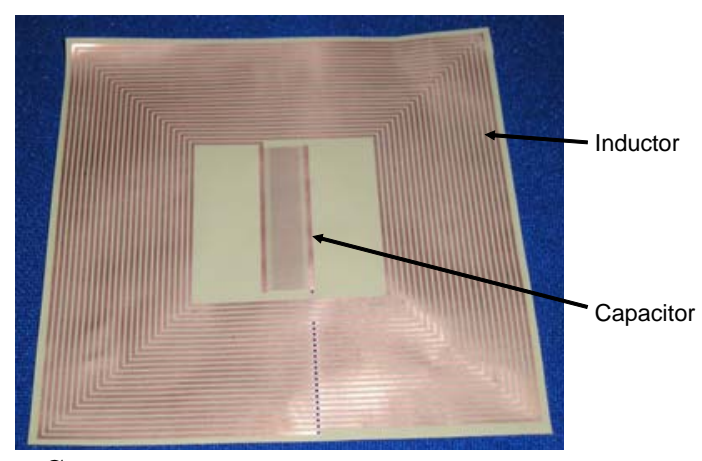

a. Sensor components

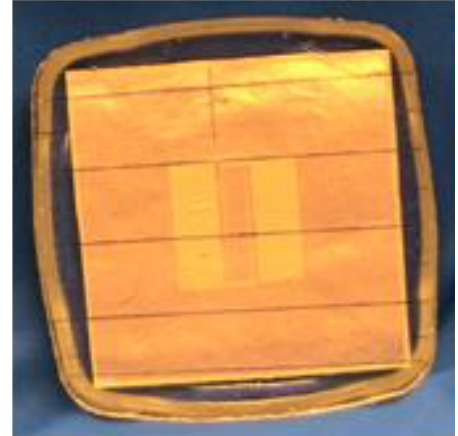

b. Sensor embedded in resin

Fig 7 Magnetic field response sensor embedded in cured resin.

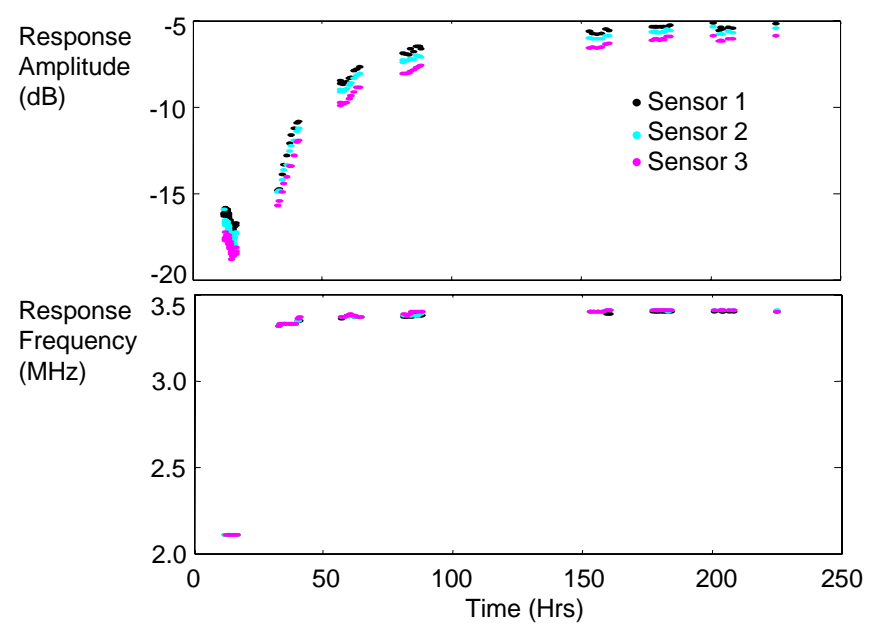

Fig 8 Time history of response amplitudes of three sensors placed in resin while it is curing. 


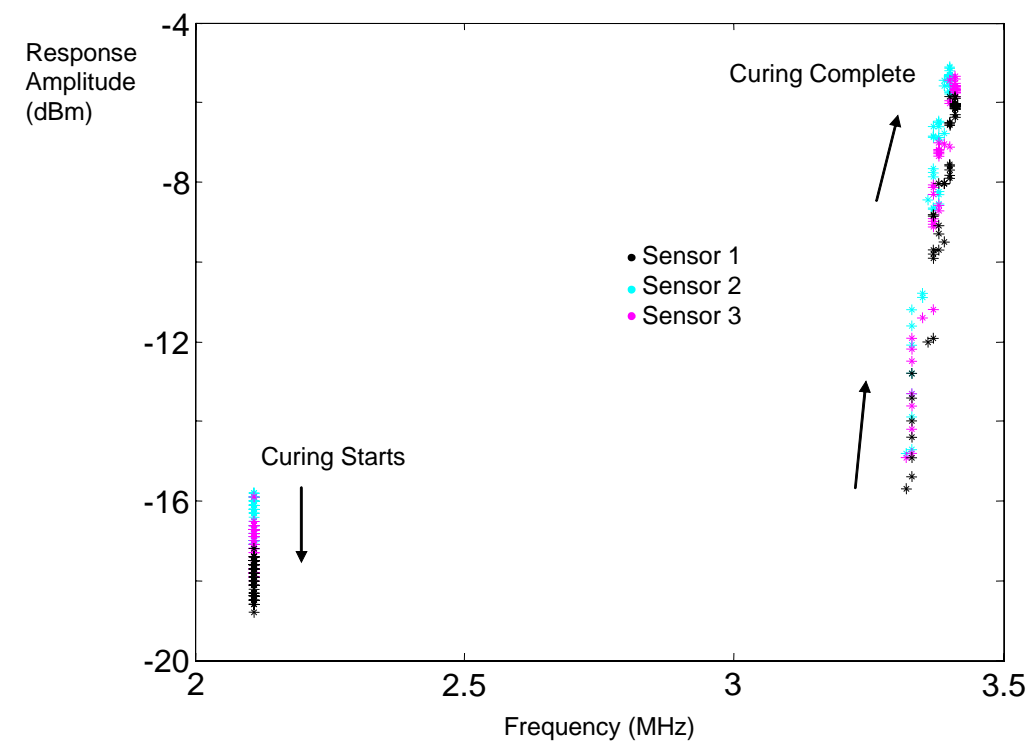

Fig 9 Amplitude-frequency response trajectories for three sensors during resin curing. 


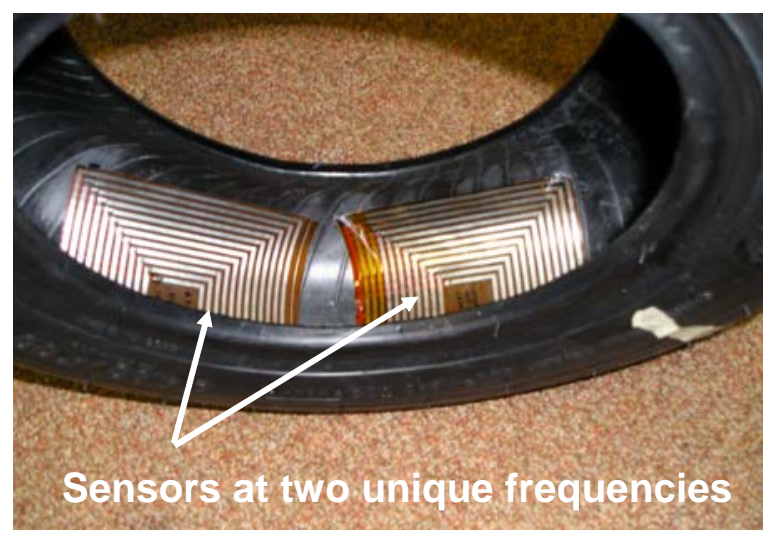

Fig 10 Two response sensors with different resonant frequencies inside of wheel

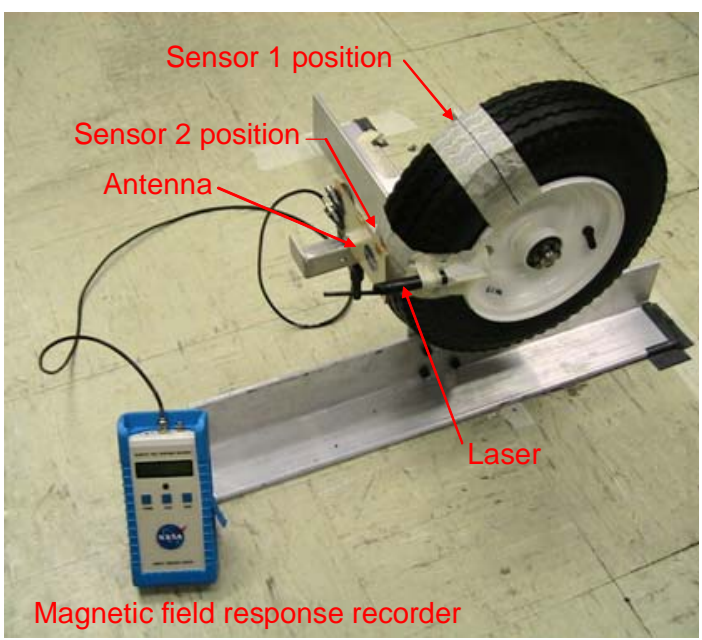

Fig 11 Measurement of wheel rotation using response recorder and two response sensors

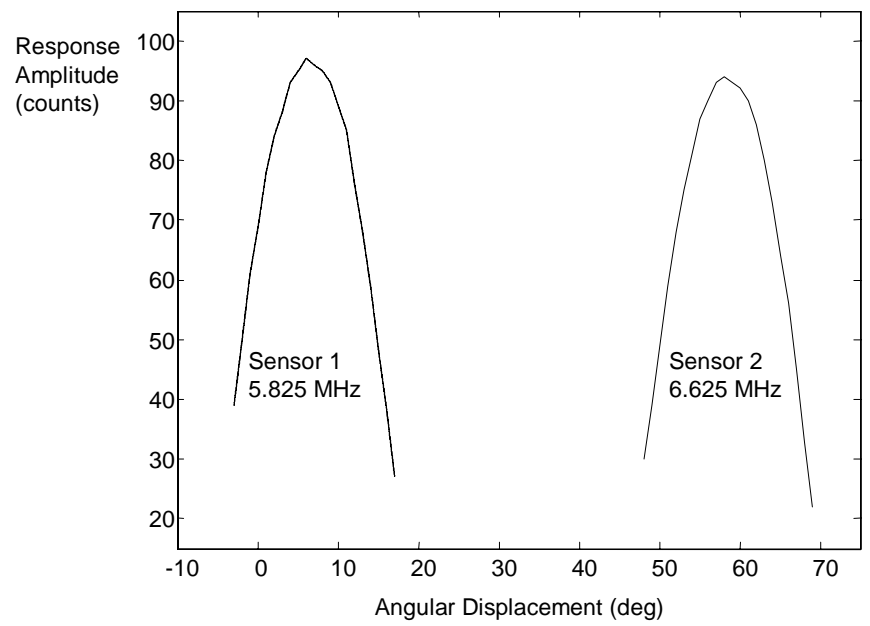

Fig 12 Response amplitude variation with rotational angle for two sensors 


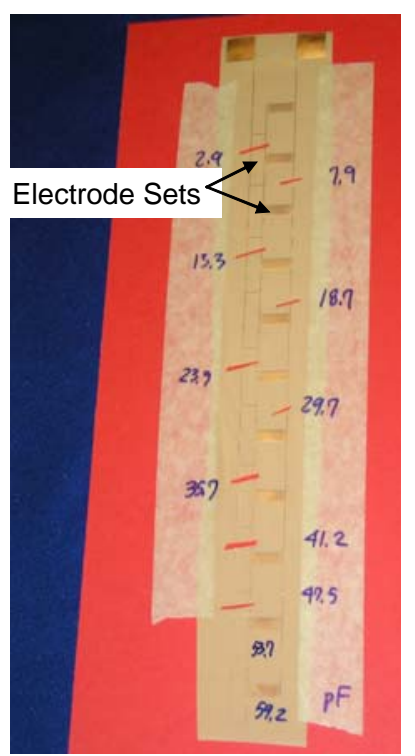

a. Electrodes of multi-measurement sensor being individually severed from circuit

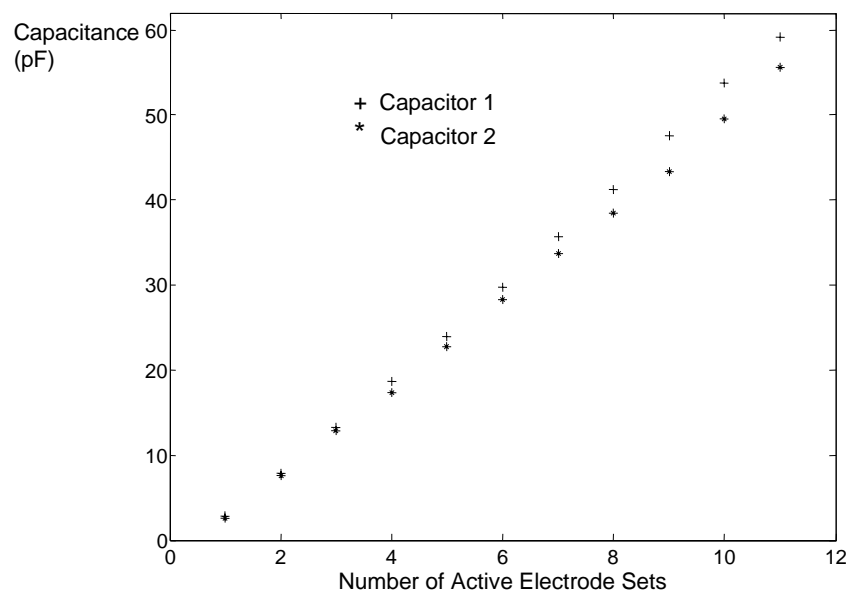

b. capacitance dependency upon number of active electrodes

Fig 13 Electrode sets of multi-measurement sensor and capacitance dependency upon number of active electrodes

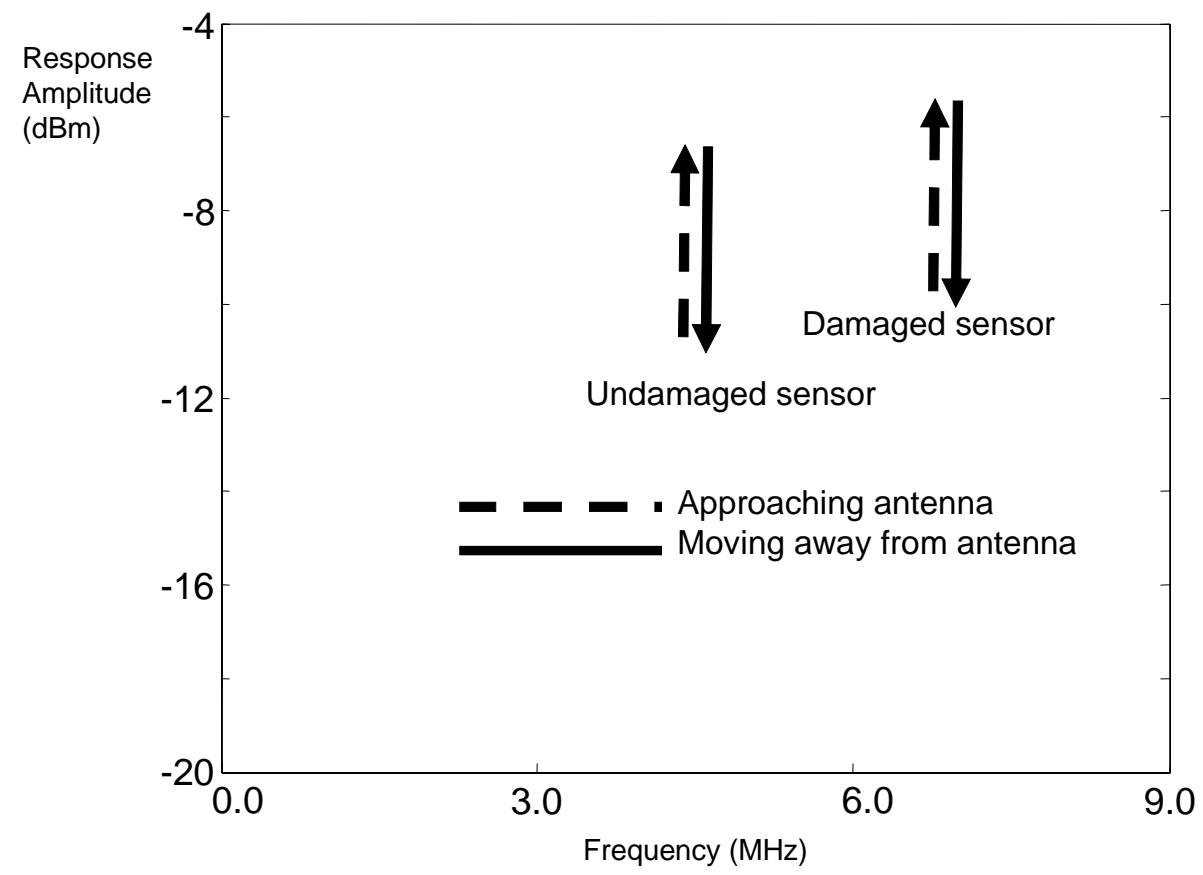

Fig 14. Amplitude-frequency response trajectory for rotation with respect to fixed antenna 